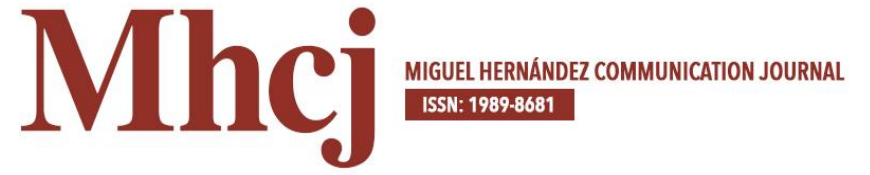

\title{
El proceso de cartelización en entornos institucionalizados: legitimación y comunicación política
}

\author{
Ddo. Gonzalo Pardo Beneyto | gonzalo.pardo@protocoloimep.com \\ Instituto Mediterráneo Estudios de Protocolo
}

Dra.María Ángeles Abellán López| ma.abellan@umh.es

Universidad Miguel Hernández

\author{
Palabras clave \\ Partido Cártel; Profesionalización; Neo- \\ institucionalismo; Comunicación Política; Democracia \\ de Audiencia; Legitimidad. \\ Sumario \\ 1. Introducción 2. El neo-institucionalismo: \\ supervivencia y legitimidad 3. La cartelización como \\ evolución de los partidos políticos 4. Democracia de \\ audiencias, pluralismo polarizado y americanización \\ de los medios de comunicación 5. Discusión 6 . \\ Conclusiones 7. Bibliografía 8. Notas.
}

\section{Resumen}

El presente trabajo tiene como objetivo principal el análisis de las tendencias cartelizadoras de los partidos políticos que se materializan en la profesionalización de los procesos comunicativos en las instituciones (Mair, 2015).

Es indiscutible el protagonismo de los medios de comunicación en la democracia de audiencia (Manin, 1998) hasta el punto que la política mediática se configura como una de los rasgos principales del espacio

público.

Los partidos políticos se han convertido en plataformas de colocación de sus líderes para que ocupen el mayor número de cargos institucionales, de forma que el fenómeno de la cartelización se ha extendido como una práctica común de nuestro sistema político. La emergencia de una élite partidista, que se ha profesionalizado en ocupar altos cargos políticos-institucionales, ha colonizado el liderazgo de las instituciones públicas y ha dado lugar a la aparición de relaciones de dependencia entre élites políticas e instituciones. Esta situación explica la necesidad constante de búsqueda de legitimidad mediante diversas estrategias, siendo una de las principales la comunicativa.

\section{Cómo citar este texto:}

Gonzalo Pardo Beneyto y María Ángeles Abellán López (2017): "El proceso de cartelización en entornos institucionalizados: legitimación y comunicación política", en Miguel Hernández Communication Journal, nº, pp. 295 a 317. Universidad Miguel Hernández, UMH (Elche-Alicante). Recuperado el _ de del artículo en mhjournal.org] de $20 \ldots$ de: $\lfloor$ link 


\section{Cartelization process in institutionalized environments: legitimation and political communication}

Ddo. Gonzalo Pardo Beneyto | gonzalo.pardo@protocoloimep.com
Instituto Mediterráneo Estudios de Protocolo

Dra. María Ángeles Abellán López| ma.abellan@umh.es Universidad Miguel Hernández

\section{Keywords}

Cartel Party; Professionalization; Neoinstitutionalism; Political Communication;

Audience Democracy; Legitimacy.

Summary

1. Introduction 2. Neo-institutionalism: survival and legitimacy 3 . The cartelization process as an evoluDtion of political parties 4. Audience democracy, polarized pluralism and Americanization of Media 5. Discussion 6. Conclussions 7. Bibliography 8 . Notes.

\section{Abstract}

The main objective of this paper is to analyze cartel party tendencies toward professionalization of communicative institutional processes (Mair, 2015).

The main role of the media in a audience democracy is indisputable (Manin, 1998). So the media politics are one of the main features of public space.

In this way, Political parties have become platforms of placement of their leaders to occupy the largest number of institutional positions. Consequently, this phenomenon has spread as a common practice of our political system. The emergence of partisan elite, which has professionalized itself in high political-institutional positions, has colonized the leadership of public institutions. It has given rise to relations of dependence between political elites and institutions. This situation explains the constant need for the search for legitimacy with strategies, one of the main being communicative.

\section{How to cite this text:}

Gonzalo Pardo Beneyto y María Ángeles Abellán López (2017): “Cartelization process in institutionalized environments: legitimation and political communication”, en Miguel Hernández Communication Journal, nº, pp. 295 to 317. Universidad Miguel Hernández, UMH (Elche-Alicante). Accessed 20_ in: [paper link in mhjournal.org] 


\section{Introducción}

Las democracias actuales están inmersas en un entorno complejo en el que se producen cambios que afectan directamente a nuestros sistemas políticos y, por ende, a los mecanismos de representación. La globalización, la aparición de las nuevas tecnologías, los riesgos medioambientales o el terrorismo son solo algunos ejemplos de fenómenos extraordinariamente complejos que están variando la forma de entender el papel del Estado-Nación en un mundo cada vez más interconectado.

Una complejidad que, sin duda, se refuerza por una "liquidez" en las instituciones ${ }^{1}$ y que aparece como un rasgo en los sujetos encargados de licuar los valores previos que tienen preferencia en la sociedad que nos rodea (Bauman, 2003). La fundamentación segura de conceptos que antaño daban solidez a las narraciones, ha dejado paso a la incertidumbre, al relativismo y, con ello, el Estado, la soberanía, la democracia han tomado una pluralidad de formas y conceptualizaciones que dependen de dos elementos fundamentales: 1) que los gobiernos sean capaces de justificar sus acciones correctamente ante la ciudadanía y, 2) que se produzca un intercambio de información constante entre medios de comunicación e instituciones político-administrativas (Rosanvallon, 2006; 2011).

Las nuevas tecnologías de la información y la comunicación han introducido cambios sustanciales y nuevas metodologías comunicativas en la esfera pública destinadas a un público global. Sin embargo, la comunicación política mediada entre medios de comunicación e instituciones políticas sigue siendo el modelo predominante en nuestras sociedades (Mazzoleni, 2010).

No en vano, estamos inmersos en lo que se ha definido por Manin (1998) como una democracia de audiencia en la que las instituciones son desplazadas por los medios de comunicación como foro de discusión y entre otros rasgos, las estrategias de marketing, el papel de los spin doctors ${ }^{2}$, la planificación mediática y los asesores del candidato forman parte fundamental del diseño de las campañas electorales y, en general, del tratamiento de los asuntos políticos.

A esto, se debe sumar una tendencia presente en todas las democracias avanzadas y que se concreta en una percepción de lejanía de la ciudadanía que se refuerza en dos fenómenos que se retroalimentan. Por un lado, la identificación entre clase social y preferencias de voto ha desaparecido casi en su totalidad por lo que clivajes tradicionales como el eje izquierda-derecha ya 
no son válidos, de forma preferente, para caracterizar la identificación partidista. De hecho, los determinantes electorales han disminuido y las clases sociales, como categorías analíticas ya no constituyen predictores fuertes de la preferencia partidista (Mair, 2015). Así, se puede afirmar que las fracturas en las que se ordena el debate partidista son mucho más complejas que antaño, fruto de la aparición de nuevos temas que obligan a los partidos y sus líderes a posicionarse sobre los mismos (van der Eijk, 1992; Franklin, 1992; Katz y Mair, 1995; Kitschelt, 2004).

Por otro, cabe destacar la falta de afiliación en los partidos políticos y de fidelidad fruto de estrategias que buscan seducir a un votante poco ideologizado y que se corresponde con el teorizado en el teorema del votante medio (Downs, 1957; Kirchkheimer, 1966; Norris, 2003). La desestructuración de los electorados tradicionales ha dejado paso a los medios de comunicación más espacio para fijar las agendas (Mair, 2015:59). Comprensiblemente, este autor se centra en el papel de los medios de comunicación tradicionales y de masas, si tenemos en cuenta que, aunque cada vez más importantes, los medios surgidos de la revolución Web 2.0 todavía están lejos, en cuanto usuarios, respecto a otros como la televisión (Martínez Sanchis, 2010).

Tal como afirman Katz y Mair (1995), se ha producido una disociación entre los partidos y la sociedad civil, situación que ha provocado que exista una relación -casi simbiótica- entre el Estado y las estructuras partidistas. El elemento clave en esta transformación es el auge del partido en el gobierno puesto que se han convertido en parte del Estado, en órganos de gobierno más que de representación. Los partidos se orientan a ocupar cargos públicos y obtener un puesto en el gobierno y esto no es solo una expectativa habitual sino también un fin en sí mismo. Como llegar al gobierno era lo importante y los programas, políticas, y estrategias de los partidos se ajustaron a este objetivo competitivo supremo (Mair, 2015).

Con todos estos precedentes, el presente artículo propone como hipótesis de trabajo que la comunicación política es la herramienta principal con la que se legitima el proceso de carterlizacion de los partidos políticos. Entendemos como cartelización de los partidos políticos de gobierno el estrechamiento de las relaciones entre estas organizaciones y las estructuras políticoadministrativas estatales con el objetivo de que los primeros se perpetúen en el poder. El planteamiento fundamental es que las instituciones partidistas utilizan los medios disponibles en las instituciones para suplir el distanciamiento que se ha producido con la sociedad civil (Katz y Mair, 1995). A su vez, las necesidades de legitimación a través de la comunicación provocan que exista un reclutamiento de personal en las instituciones. 
Para desarrollar los principales argumentos que conformarán estas dos hipótesis de trabajo interconectadas, se utilizará el enfoque neoinstitucionalista como el marco teórico que sostiene la influencia de las instituciones en las decisiones individuales puesto que modelan las diversas preferencias políticas. Una vez, expuesta esta influencia, se explicará el proceso de cartelización de los partidos políticos y su influjo en el sistema político español; así como sus vinculaciones con los medios de comunicación. Bajo el telón de fondo de este planteamiento teórico, profundizaremos en las hipótesis de investigación para exponer en una discusión teórica, sin ánimo de exhaustividad, sobre los contenidos abordados. Por último, comentaremos las principales líneas de investigación relacionadas en una conclusión.

\section{El neo-institucionalismo: supervivencia y legitimidad}

Se podría afirmar que el último tercio del siglo XX ha sido la era del "Homo Economicus", en el que la racionalidad económica y la maximización del beneficio fueron $-\mathrm{y}$ en algunas partes siguen siendo- los valores centrales de la sociedad. Dichas tendencias tuvieron sus ramificaciones a nivel institucional con la introducción de técnicas basadas en la eficiencia procedentes de la Nueva Gestión Pública y en el plano político con ideologías como el Neoliberalismo. Desde un punto de vista académico, el interés se materializó en estudios centrados en las decisiones del individuo y la cuantificación de preferencias (Held, 2006; Hood, 1991; Shepsle y Bonchek, 1997).

Sin embargo, esta tendencia economicista ha sido cuestionada desde diferentes posiciones produciéndose un giro hacia lo institucional. Las ciencias sociales han recuperado un enorme interés por el papel que desempeñan las instituciones en las sociedades contemporáneas y cómo afectan a los individuos en la toma de decisiones y en sus comportamientos (Ostrom, 1999). No en vano, las políticas de innovación política tienen un claro carácter empoderador y de apertura de las instituciones públicas, así como económicas (Canales Aliende, 2014).

El neo-institucionalismo pone el foco en las instituciones, en sus reglas, procedimientos y sistemas culturales y, como enfoque teórico, nos permite estudiar las instituciones y la forma en la que estas se relacionan con su entorno. En este trabajo se considera que las instituciones incorporan procesos y formas de actuar con el objetivo de dar contestación a aquello que espera su entorno inmediato ${ }^{3}$. Este proceso se concreta en distintas estrategias marcadas por el isomorfismo coercitivo, el isomorfismo mimético y el 
isomorfismo normativo4 (DiMaggio y Powell, 1983; Meyer y Rowan, 1977; Peters, 2005).

Además, no se puede olvidar que estos cambios no son automáticos, ya que los individuos dentro de una institución son los que deben aceptar o censurar las nuevas prácticas utilizadas en base a un comportamiento estratégico ${ }^{5}$ (DiMaggio, 1988; Oliver, 1991).

¿Pero qué es lo que buscan las instituciones del entorno? Básicamente, buscan generar legitimidad puesto que sin ella no puede persistir ningún sistema político. Max Weber (2002), la definía como la justificación de la que se sirve el poder político para fundamentar su autoridad y de que un determinado orden político sea aceptado pacíficamente. Por su lado, Lipset (2001) entendía la legitimidad como la capacidad del sistema para mantener la creencia en el funcionamiento de las instituciones políticas como las más adecuadas para la sociedad.

En base a estas concepciones y desde un punto de vista neo-institucional, estrictamente, puro, podemos definir la legitimidad como: "[...] a generalized perception or assumption that the actions of an entity are desirable, proper, or appropriate within some socially constructed system of norms, values, beliefs, and definitions" (Suchman, 1995: 574). Por lo que las prácticas y los cambios organizativos puestos en marcha en las organizaciones de un sector determinado son adoptadas con el objetivo de generar legitimidad hacia las instituciones (Tolbert y Zucker, 1983; Leblebici et al. 1991).

La legitimidad se convierte en un término con múltiples acepciones que se materializan en distintos ámbitos de legitimadores (Bitektine, 2011). En primer lugar, encontramos cómo las instituciones necesitan estar conforme con las normas legales o cuasi-legales de un territorio. En segundo lugar, éstas han de adaptarse a los valores sociales y culturales básicos de una sociedad. Por último y relacionado íntimamente con la percepción del individuo, han de tener su aceptación directa (Scott, 1995).

Esta última acepción está íntimamente conectada con aquello que se percibe, así como con los elementos de juicio del individuo y su clasificación valorativa de lo que un acto, una acción, un objeto o un valor es positivo o negativo para sí mismo y la sociedad que lo rodea (Bourdieu, 1997). La legitimación es, de esta forma, un valor percibido que se relaciona directamente con elementos intangibles y socialmente construidos que para las organizaciones son recursos a gestionar y tener en cuenta (Oliver, 1997). 
El enfoque neo-institucionalista nos proporciona el utillaje conceptual para captar más afinadamente la importancia de la legitimidad en un contexto de cambios e intercambios entre partidos políticos, élites partidistas e instituciones. Y es que la profesionalización de las élites y de las burocracias de los partidos, -con gran especialización y sectorialización de sus órganos centrales-, refuerza la idea de una clase política que vive exclusivamente de ella de manera profesional.

\section{La cartelización como evolución de los partidos políticos}

Los partidos políticos constituyen piezas clave del sistema representativo puesto que cumplen con una serie de funciones tradicionales importantes tanto para la sociedad en la que se incardinan como para las instituciones a las que quieren acceder. Así y tal como afirma Alcántara (1997) son los encargados de: a) transmitir valores políticos; b) movilizar ante problemáticas concretas; c) canalizar las formas de participación política convencional; d) legitimar la representación política a través de un sistema de elecciones libres; e) representar a la ciudadanía en las instituciones y; e) producir políticas públicas viables. Además, de seleccionar líderes y candidatos políticos (Ware, 1996).

Aunque puede parecer que los partidos políticos son organizaciones poco dadas a modificar su organización y funcionamiento, lo cierto es que a lo largo de la historia han sufrido una serie de cambios profundos en la relación que tienen con la sociedad civil y las instituciones. Tanto es así que el declive de las identidades partidistas es uno de los cambios más reveladores que se han producido en la política de masas europea en los últimos 30 años (Mair, 2015).

La aparición de los partidos políticos de cuadros fue una reacción racional a un parlamentarismo que se basaba en la exclusividad de la representación política. Con la ampliación del sufragio, aparecen en la escena institucional los partidos de masas, lo que supuso la incorporación de facto de los intereses de los trabajadores en las instituciones representativas. Ya a mediados del siglo $\mathrm{XX}$, se produce otra transformación y es que estos partidos que representaban a las clases sociales presentes en un Estado-Nación concreto (Manin, 1998) se convierten en "partidos atrapalotodo" (Kirchkheimer, 1966). Esta expresión caracteriza a las organizaciones partidistas que buscan ampliar al máximo su base electoral interclasista, tienden hacia el pragmatismo político con la adopción de programas políticos flexibles y con menor ortodoxia ideológica para captar el mayor número de votos. 
El atrapalotodo es un modelo competitivo que acabó con los fuertes vínculos de representación para lograr más éxito electoral. Estos partidos perseguían llegar al gobierno y ocupar puestos en las instituciones y administraciones públicas, por encima de la representación. Como llegar al gobierno era lo importante, los programas, las políticas y las estrategias de los partidos se ajustaron a este objetivo competitivo supremo. Las implicaciones de esta afirmación suponen que lo importante es conseguir el voto y no tanto la educación política de la ciudadanía (van der Eijk, 1992; Downs, 1957; Franklin, 1992; Katz y Mair, 1995; Kirchkheimer, 1966; Kitschelt, 2004; Mair, 2015).

De forma sumaria, podemos destacar que los ejes fundamentales de los partidos cártel son los siguientes: a) los partidos en el gobierno definen aspectos capitales del sistema electoral, la regulación de las organizaciones partidistas, la financiación y la comunicación institucional; b) las relaciones que se producen dentro del propio partido que derivan en la profesionalización de la política, la existencia de asesores profesionalizados y en una estructura de estratarquía en la que se reproducen los patrones de la organización central del partido; c) en la competencia en la arena electoral y los mensajes que se lanzan al público con un claro carácter centrípeto con el objetivo de conservar el statu quo y; d) en el comportamiento profesionalizado de los representantes del partido en el desempeño de cargos públicos políticoadministrativos (Detterbeck, 2005; Hutcheson, 2012; Kopecky y Mair, 2011; Sandri y Pauwels, 2010).

A modo de resumen y tal como se muestra en el diagrama 1 se produce una relación estrecha entre los Partidos Políticos y las Instituciones, en la que los partidos se convierten en un bróker de los intereses de la sociedad civil. A este hecho se debe sumar la lejanía de los partidos políticos con la sociedad civil (Katz y Mair, 1995).

Diagrama 1. La relación entre los partidos políticos cártel, el Estado y la sociedad civil Fuente: Adaptado de Katz y Mair, 1995. 

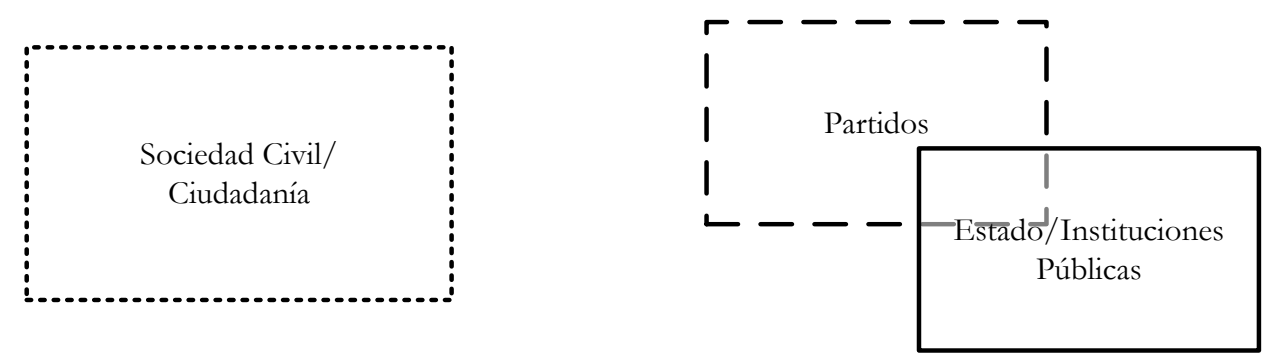

Parte del debate teórico se ha centrado en discutir la validez de la tesis del partido cártel en la sociedad. Autores como Kitschelt (2004) achacan la distancia entre partidos y sociedad civil por la quiebra que se produce en los clivajes tradicionales y añade un componente liberal y autoritario en sus posiciones. Asimismo, Koole (2004) destaca el carácter independiente de los medios de comunicación y los movimientos sociales y su posición eminentemente predominante para obligar a los partidos políticos a adoptar medidas democratizadoras o que fomentan la transparencia y la participación como las primarias.

\section{Democracia de audiencias, pluralismo polarizado y americanización de los medios de comunicación}

Los medios de comunicación son un elemento esencial para la democracia, ya que estos son importantes para la formación de una opinión pública libre o, lo que es lo mismo, la capacidad que tiene el individuo para poder opinar en libertad sobre los asuntos públicos (Sartori, 1992). Al mismo tiempo, actúan como la arena en la cual los distintos contendientes políticos confrontan los diferentes mensajes enfrentados en pro de concentrar la atención mediática y ciudadana que es mucho más intenso en unos comicios electorales (Martín Salgado, 2006).

Aceptado el rol de los medios y de la necesidad de fuentes alternativas de información en una sociedad democrática, a mediados de los años noventa, Bernard Manin (1998) difundió la expresión democracia de audiencia para referirse, según su interpretación, a la tercera gran transformación del gobierno representativo. Esta transformación se caracterizaba por un protagonismo muy destacado de los líderes políticos, una ciudadanía pasiva, la voracidad electoral de los partidos políticos y la dependencia de la democracia de los medios de comunicación. Todos estos fenómenos han debilitado la relación entre representantes y representados. 
En este nuevo escenario, los representantes políticos son conocedores del éxito que reporta una correcta escena mediática y los discursos políticos que buscan legitimar el status quo, se ajustan a las necesidades y requerimientos comunicativos. Por tanto, se generan discursos para proporcionar un titular que impacte en la opinión; discursos, pues, que digan poco y no expliquen nada. Igualmente, la racionalidad política convencional se está transformando en este nuevo espacio público mediatizado. Una de las más importantes es la visibilidad de los acontecimientos políticos porque lo que es visible pasa a ser dominio público. Como la comunicación mediática es inseparable del sistema político, los argumentos estrictamente políticos acaban por adecuarse a los imperativos mediáticos puesto que, sin visibilidad, no se difunden las noticias ni se llega a la ciudadanía (Ortega, 2011).

Aun así, no deja de ser paradójica la relación que existe entre estas organizaciones y las democracias liberales actuales si atendemos a la idea de que estamos inmersos en una democracia de audiencia. En ella, los medios de comunicación no son solo un actor más, sino que también son el ágora política en la que se canalizan las discusiones públicas y en la que se produce una confrontación de opiniones en base a fracturas programáticas existentes (Mazzoleni, 2010), tal como se muestra en el diagrama 2.

Esta situación ha derivado en una proliferación de profesionales de la política que se dedican a asesorar sobre temas de comunicación a nivel de partido e institucional (Manin, 1998). Un fenómeno que no es algo desdeñable si atendemos a la idea de que el acto comunicativo es, por sí mismo, una muestra de poder, en tanto en cuanto los medios de comunicación configuran el pensamiento del individuo (Castells, 2009).

Diagrama 2. La relación entre los medios de comunicación, la ciudadanía, las instituciones y la política en el modelo de comunicación mediada Fuente: Adaptado de Mazzoleni (2010) 


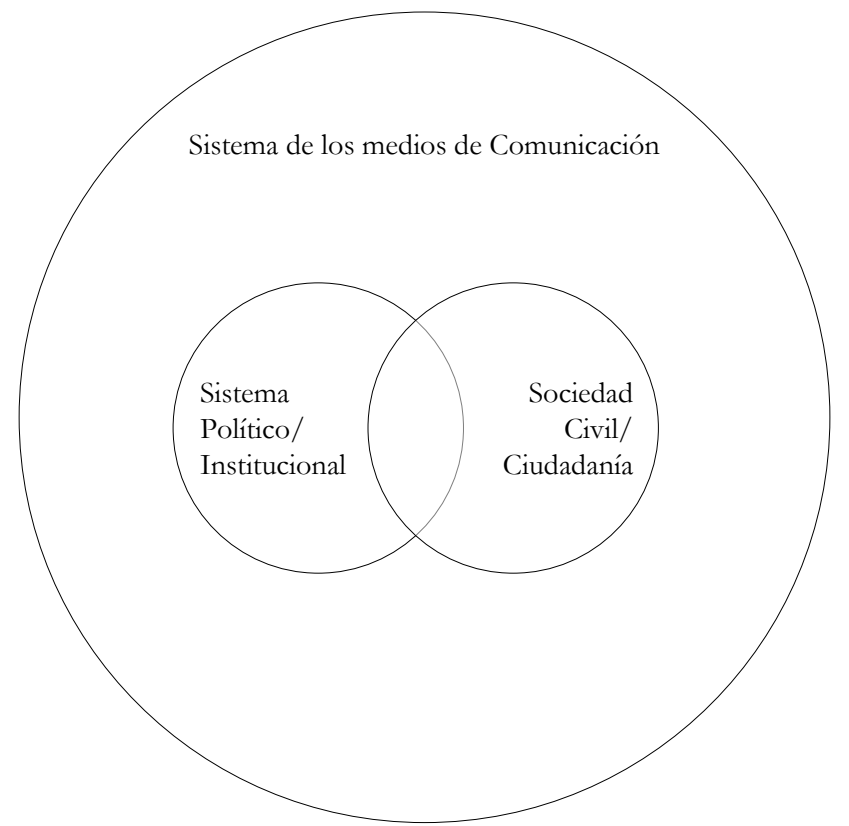

Este escenario parece conectarse directamente con lo que se ha llamado por algunos autores como "americanización"7 (Mancini y Swanson, 1996) de la comunicación política que no es otra cosa que la asimilación de las prácticas norteamericanas en estos menesteres. Así se produce un uso del marketing, la hipermediatización, la personalización de la política y la consecuente profesionalización del mensaje político e institucional (Sánchez Medero, 2009). A esto, se debe a que los medios de comunicación son, en su gran mayoría, empresas y lo que buscan, por tanto, es maximizar el beneficio obtenido (Lippman, 2003; Ortega, 2011).

En el caso español, además, estamos inmersos en lo que se ha denominado un sistema mediático de pluralismo polarizado (Hallin y Mancini, 2008). Esto significa que se parte de una situación en la que: a) no existe un desarrollo del mercado de la prensa escrita al igual que ocurre en los países del norte de Europa y en los anglosajones; b) no se produce una profesionalización efectiva de los periodistas hasta bien entrado el siglo XX c) existe una conexión directa entre el periodismo, la política y los empresarios de los medios, que viene auspiciado por un control político indirecto de los mensajes mediáticos a través de subvenciones y publicidad institucional; d) se da una fusión entre comentarios e información política; e) concurre el uso partidista de los medios de radiotelevisión pública; f) las sociedades profesionales de periodistas no tienen fuerza para instituirse en un contrapoder; g) no hay una demanda de medios de comunicación escritos y; h) no hay una regulación interna que dote 
de preeminencia a los medios de comunicación. Por esta razón, se produce un déficit en el mercado de medios que es mitigado por el Estado a través de la entrega de grandes sumas de dinero público para publicar anuncios y otros tipos de materiales publicitarios; situación que ha dado lugar a la influencia del político en los medios (Hallin y Mancini, 2008; Innerarity, 2006; Martínez Sanchis, 2010).

Sin ninguna duda, este panorama colisiona directamente con las posturas de Koole (2004) y Kitschelt (2004) ante la tesis del partido cártel y en las que se daba una preeminencia a los movimientos sociales y los medios de comunicación. Esto se explica por la relación que existe entre política y estos últimos a través de los accionistas y su influencia en las informaciones al no existir instituciones que velen por el rigor periodístico y la separación entre comentario e información (Hallin y Mancini, 2008) ${ }^{8}$.

\section{Discusión}

Tanto los procesos de legitimación como el fenómeno de los partidos cártel, así como el pluralismo polarizado y la americanización de la comunicación política son elementos teóricos importantes para fundamentar las relaciones que se producen entre la sociedad civil/ciudadanía, los partidos y las instituciones públicas en su conjunto.

Teniendo en cuenta lo expuesto, hasta el momento, pensamos que estas teorías conjunta e interconectadamente pueden dar respuesta a las siguientes hipótesis, a través de un modelo teórico a aplicar en el caso español, tal como se muestra en el Diagrama 3:

\section{Diagrama 3. Sistema de relaciones de un partido cártel en una democracia de audiencias Fuente: Elaboración propia.}




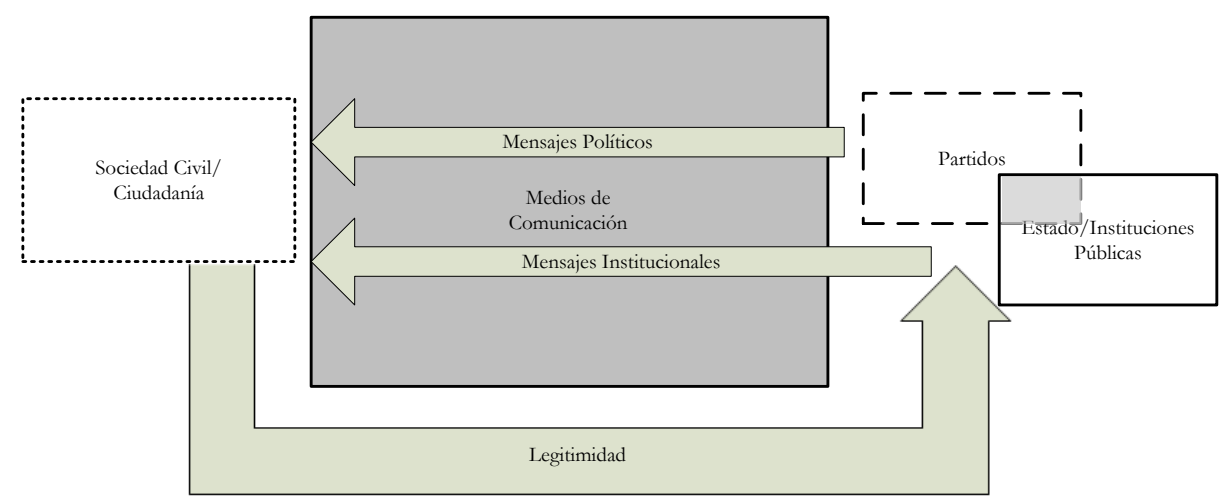

En primer lugar, debemos hacer mención a la relación que se produce entre el Estado/instituciones y los partidos con la sociedad civil/ciudadanía. Como ya se ha mencionado, existe un evidente alejamiento entre representantesgobernantes de los representados-gobernados. El partido cártel en sí mismo, se convierte en un elemento de espectáculo, imagen y teatro y los medios son un elemento fundamental que caracteriza las relaciones entre la ciudadanía y la política (Katz y Mair, 2009). De esta forma, ocupan el papel de correa de transmisión de lo que ocurre en las instituciones y el ámbito político hacia la ciudadanía (Mazzoleni, 2010). La legitimación se produce top down, es decir, las élites y las instituciones se legitiman a sí mismas hacia abajo, buscando la aprobación de la masa crítica ciudadana.

Pero los protagonistas políticos de las crónicas no se centran en buscar que haya una ciudadanía informada (Sartori, 1992), sino que ponen en marcha estrategias encaminadas a explotar sus fracturas con el objetivo de legitimar su imagen y, por tanto, su acción de gobierno (Manin, 1998; Rao, 1994).

De forma inversa, los medios de comunicación son parte de las relaciones comunicativas maximizando los elementos espectaculares e informativos, práctica que es utilizada por los profesionales de la comunicación política para explotar las fracturas y la figura de los políticos ${ }^{9}$ con el objetivo de incrementar las ventas de su compañía y, por tanto, sus beneficios (Innerarity, 2006; Martín Salgado, 2006).

Por lo que aquello que se comunica a una sociedad civil y una ciudadanía alejada de sus instituciones es cada vez más importante para salvaguardar la perpetuación política de los actores políticos en las instituciones (Meyer y Rowan, 1977; Katz y Mair, 1995, 1996, 2009). Una situación que tiene posibilidades de maximizarse e intensificarse por parte de los partidos políticos a través de los instrumentos del Estado en un sistema de medios de 
comunicación basado en el pluralismo polarizado en el que 1) por una parte, no se distingue entre información y comentario y, 2) por otra, existe un bajo grado de profesionalización al no existir organizaciones corporativistas de periodistas y al haber profesionales de otras áreas de conocimiento (Hallin y Mancini, 2008).

Ante esta dinámica comunicativa podemos formular la siguiente hipótesis de trabajo:

H1: La comunicación política es la herramienta principal con la que se legitima el proceso de cartelización de los partidos políticos.

La necesidad de comunicar de forma efectiva por parte de los partidos y las instituciones, produce lo que ha sido llamado por autores como Panebianco (2009) la profesionalización de los principales actores políticos. Este efecto no solo se circunscribe a los miembros electos de un partido cártel (Katz y Mair, 1996), sino que afecta directamente a los asesores y al personal directivo dependiente del cargo electo (Gómez y Verge, 2012).

Partiendo de esta definición y del entorno que envuelve a las instituciones y partidos políticos podemos afirmar que aquellos puestos que sean de libre disposición tendrán un alto porcentaje de profesionales de la comunicación en su seno. Esto se explica por la relación que se produce entre el partido y las instituciones ya que esta se estrecha. Provoca, por tanto, una situación en la que se produce un control de los recursos del Estado por parte de la organización partidista. Esta, en última instancia, lleva a la producción de un fenómeno de patronazgo que puede ser definido como: “[...]an increasingly valuable resource with which parties can seek to ensure their organizational survival and success in contemporary political systems"10 (Kopecky y Mair, 2011:10).

Respecto a los resortes institucionales creados por el turnismo entre las distintas formaciones políticas que se encuentran dentro del cártel, hay una asignación de cargos de libre disposición como recursos de salvaguarda que, en última instancia, sirven para contestar a las necesidades del entorno (DiMaggio y Powell, 1983). Para ello, se necesitan asesores y personal directivo dedicados al proceso de mejora de la legitimidad. De esta forma, planteamos una segunda hipótesis:

H2: Las necesidades de legitimidad impulsan a los líderes de los partidos a reclutar personal experto en comunicación para incorporarlo a las instituciones políticas. 
De esta forma, las élites de los partidos se convierten en protagonistas de la dirección del país puesto que dan legitimidad a las actuaciones gubernamentales e institucionales que trazan por donde se encamina esa comunidad política. Como ya se ha hecho referencia a lo largo de estas líneas, las organizaciones partidistas necesitan recursos para perpetuarse en el poder. Estos no son solo pecuniarios, sino que los puestos disponibles en una institución sirven para cohesionar el partido, gestar una carrera profesional política, generar expectativas y, por último, crear una clase de expertos propios en temas comunicativos y político-públicos que se insertan en una formación determinada (von Beyme, 1995; Kopecky y Mair, 2011; Panebianco, 2009).

\section{Conclusiones}

Este trabajo se ha centrado en la formulación de un marco teórico y de dos hipótesis de trabajo, a partir de la conjunción del enfoque neo-institucionalista y la tesis del partido cártel como evolución del partido atrapalotodo.

Las principales ideas desarrolladas conducen a pensar que existe un planteamiento teórico e indicios suficientes para testear estas hipótesis con garantías teórico-prácticas. Asimismo, tenemos la certeza de que, ante nosotros, se encuentra un campo prolífico para analizar las relaciones comunicativas conjuntamente con la realidad partidista desde diferentes perspectivas multidisciplinares, en el contexto del panorama de medios de comunicación español.

Las conexiones entre el marco teórico desarrollado y la dinámica interna de los partidos políticos parecen prometedoras para futuras investigaciones y de manera destacada, la tesis de partido cártel y su relación directa con el papel de los cargos orgánicos del partido y su relevancia mediática.

Finalmente, se podrían realizar estudios que tuviesen en cuenta la estrategia de los partidos cártel tradicionales en la defensa de sus intereses legítimos respecto a las nuevas formaciones. Tampoco hay que olvidar una relación de actualidad en los últimos tiempos que liga el neo-institucionalismo con el comportamiento de los partidos políticos, la corrupción y los procesos de comunicación política. 


\section{Bibliografía}

Alcántara, M. (1997). Las tipologías y funciones de los partidos políticos. En M. Mella Márquez (Ed.), Curso de Partidos Políticos (pp. 37-57). Madrid: Akal.

Bauman, Z. (2003). Modernidad Líquida. México D.F.: Fondo de Cultura Económica.

von Beyme, K. (1995) La clase política en el Estado de Partidos. Madrid: Alianza.

Bitektine, A. (2011). Toward a theory of social judgements of organizations: the case of legitimacy, reputation, and status. Academy of Management Review 36, Nueva York, enero, pp. 151-179.

Bourdieu, P. (1997). Razones prácticas. Sobre la teoría de la acción. Barcelona: Editorial Anagrama.

Canales Aliende, J.M. (2014). Cambio Institucional, Liderazgo y Políticas Públicas. En G. Pastor Albaladejo (Ed.), Teoría y Práctica de las Políticas Públicas (pp. 455-477). València: Tirant Lo Blanch.

Castells, M. (2009). Comunicación y Poder. Madrid: Alianza.

Detterbeck, K. (2005). Cartel parties in Western Europe? Party Politics 11, Londres, marzo, pp.173-191.

DiMaggio, P. (1988). Interest and Agency in Institutional Theory. En L. G. Zucker (ed.), Institutional pattems and organizations: Culture and environment (pp. 321). Cambridge, MA: Ballinger.

DiMaggio, P. y Powell, W.W. (1983). The Iron Cage Revisited: Institutional Isomorphism and Collective Rationality in Organizational Fields. American Sociological Review 48, Washington D.C, abril, pp. 147-160.

Downs, A. (1957). An economic theory of political action in a democracy. Journal of Political Economy 65, Chicago, abril, pp. 135-150.

van der Eijk, C. et al. (1992). Cleavages, conflict resolutions and democracy. En VVAA, Electoral Change (pp. 406-431). Cambridge: Cambridge University Press. 
Franklin, M. (1992). The decline of cleavage politics. En VVAA, Electoral Change (pp. 383-405). Cambridge: Cambridge University Press.

Gómez, R. y Verge, T. (2011). Party Patronage in Spain: Appointments for Party Government. En P. Kopecky, P. Mair y M. Spirova (Eds.), Party Patronage and Party Government in European Democracies (pp. 3-16). Oxford: Oxford University Press.

Greenwood, R.; Oliver, C.; Sahlin, K. y Suddaby, R. (2008). Introduction. En VV.AA (Eds.), The Sage Handbook of Organizational Neo-Institutionalism (pp. 146). Thousand Oaks: Sage.

Held, D. (2006). Models of Democracy. Cambridge: Polity Press.

Hood, C. (1991). A Public Management for all Seasons? Public Administration 69, Nueva Jersey, marzo, pp.3-19.

Hutcheson, D. S. (2012). Party cartels beyond Western Europe: Evidence from Russia. Party Politics 19, Londres, noviembre, pp. 907-924.

Katz, R.S. y Mair, P. (1995). Changing Models of Party Organization and Party Democracy: The Emergence of the Cartel Party. Party Politics 1, Londres, enero, pp. 5-28.

- (1996). Cadre, Catch-All or Cartel?: A Rejoinder. Party Politics 2, Londres, julio, pp. 525-534.

- (2009). The Cartel Party Thesis: A Restatement. Perspectives on Politics 7, Cambridge, diciembre, pp. 754-766.

Kirchkheimer, O. (1966). The Transformation of the Western European Party System. En J. LaPalombara y M. Weiner (eds.), Political Parties and Political Development (pp.177-200). Nueva Jersey: Princeton University Press.

Kitschelt, H. (2004). Diversificación y reconfiguración de los sistemas de partidos de las democracias postindustriales. Revista Española de Ciencia Politica 10, Madrid, abril, pp. 9-51.

Koole, R. (2004). ¿Partidos de cuadros, catch-all o cartel? El concepto de partido cartel. Zona Abierta 108-109, Madrid, sin definir, pp. 43-65. 
Kopecky, P. y Mair, P. (2011). Party Patronage as an Organizational Resource. En P. Kopecky, P. Mair y M. Spirova (Eds.), Party Patronage and Party

Government in European Democracies (pp. 3-16). Oxford: Oxford University Press.

Innerarity, D. (2006). El Nuevo Espacio Público. Madrid: Espasa.

Hallin, D.C. y Mancini, P. (2008). Sistemas mediáticos comparados. Tres modelos de relación entre los medios de comunicación y la política. Barcelona: Hacer.

Leblebici, H.; Salancik, G. R.; Copay, A. y King, T. (1991). Institutional Change and the Transformation of Interorganizational fields. Administrative Science Quarterly 36, Nueva York, septiembre, pp. 333-363.

Lippman, W. (2003) La opinión pública. San Lorenzo de El Escorial: Langre.

Lipset, S. M. (2001). Algunos requisitos sociales de la democracia: desarrollo económico y legitimidad política. En A. Batlle (Ed.), Diez textos básicos de Ciencia Politica (pp. 113-150). Barcelona: Ariel.

Mair, P. (2015) Gobernando el vacío. La banalización de la democracia occidental. Madrid: Alianza.

Mancini, P. y Swanson, D.L. (1996). Patterns of Modern Electoral Campaigning and Their Consequences. En D. L. Swanson y P. Mancini (Eds.), Politics, Media and Modern Democracy (247-276). Londres: Westport: Praeger.

Manin, B. (1998). Modelos de Democracia. Madrid: Alianza.

Martín Salgado, L. (2006). Elecciones y medios de comunicación: el ser y el deber. En J. Molins y P.Oñate (Eds.), Elecciones y comportamiento electoral en la España multinivel (pp.223-239). Madrid: CIS.

Martínez Sanchis, F. (2010). Periodisme local i comarcal: la comunicació valenciana de proximitat. València: Universitat de València.

Mazzoleni, G. (2010). La Comunicación Política. Madrid: Alianza.

Meyer, J.W. y Rowan, B. (1977). Institutionalized Organizations: Formal Structure as Myth and Ceremony. American Journal of Sociology 83, Chicago, septiembre, pp. 340-363. 
Norris, P. (2003). Democratic Phoenix: Reinventing Political Activism. Cambridge: Cambridge University Press.

Oliver, C. (1991). Strategic responses to institutional processes. Academy of Management Review 16, Nueva York, enero, pp. 145-179.

- (1997). Sustainable Competitive Advantage: Combining Institutional and Resource-Based Views. Strategic Management Journal 18, Nueva Jersey, octubre, pp.697-713.

Ortega, F. (2011). La Politica Mediatizada. Madrid: Alianza.

Ostrom, E. (1999). Institutional Rational Choice: An Assessment of the Institutional Analysis and Development Framework. En P. A. Sabatier (ed.), Theories of the Policy Process (pp. 35-71). Boulder: Westview Press.

Panebianco, A. (2009). Modelos de Partido: organización y poder en los partidos politicos. Madrid: Alianza.

Peters, B.G. (2005). Institutional Theory in Political Science. The New Institutionalism. Londres: Pinter.

Rao, H. (1994). The social construction of reputation: certification contests, legitimaqtion, and the survival of organizations in the American automobile industry: 1895-1912. Strategic Management Journal 15, Chicago, diciembre, pp. 29-44.

Rosanvallon, Pierre. (2006). Democracy Past and Future. Nueva York: Columbia University Press.

- (2011). Democratic Legitimacy. Impartiality, Reflexivity, Proximity. New Jersey: Princeton University Press.

Sánchez Medero, R. (2009). La comunicación política española, ¿un modelo propio o una adaptación del modelo estadounidense? Revista de Ciencias Sociales 1, Maracaibo, enero, pp. 9-23.

Sandri, G. y Pauwels, T. (2010). Party Membership Role and Party Cartelization in Belgium and Italy: Two Faces of the Same Medal? Politics and Policy 38, México D.F., diciembre, pp. 1237-1266.

Sartori, G. (1992). Elementos de Teoría política. Madrid: Alianza. 
Scott, W.R. (1995). Institutions and Organizations. Londres: Sage.

Shepsle, K. A. and Bonchek, M. S. (1997). Analyzing politics: rationality, behavior, and institutions. New York: W.W. Norton.

Suchman, M.C. (1995) Managing Legitimacy: Strategic and Institutional Approaches. Academy of Management Review 20, Nueva York, julio, pp. 571-610.

Tolbert, P.S. y Zucker, L. (1983). Institutional Sources of Change in the Formal Structure of Organizations: The Diffusion of Civil Service Reform, 1880-1935. Administrative Science Quarterly 28, Nueva York, marzo, pp. 22-39. Ware, A. (1996). Partidos políticos y sistemas de partidos. Madrid: Istmo. Weber, M. (2002). Economía y Sociedad. México D.F: Fondo de Cultura Económica.

\section{Notas}

${ }^{1}$ El término institución utilizado en este artículo es similar al que utiliza Scott (1995: 33): "Las instituciones consisten en estructuras y actividades cognitivas, normativas y reguladoras que proporcionan estabilidad y significado al comportamiento social. Las instituciones son transportadas por diversas culturas, estructuras y rutinas de transporte, y operan en múltiples niveles de jurisdicción. En esta conceptualización, las instituciones son sistemas multifacéticos que incorporan sistemas simbólicos construcciones cognitivas y normas normativas y procesos regulativos llevados a cabo a través del comportamiento social y modelado".

${ }^{2}$ El término Spin Doctor hace referencia al asesor que desarrolla su actividad entre la esfera política y comunicativa. Desde esta posición de enlace centra su labor en la conformación de mensajes que sean atractivos para la opinión pública (Mazzoleni, 2010).

${ }^{3}$ En concreto, las instituciones, sea cual sea su procedencia, ponen en marcha procesos y formas de actuar centradas en la recuperación y mejora de la legitimidad de la institución. Siguiendo a Suchman (1995) podemos afirmar que algunos de estos se centran en: 1) Posicionar la organización en un 
régimen institucional existente; 2) poner en marcha procesos reactivos de cambio que no se confronten con el entorno actual; 3) manipular el contexto con el objetivo de conseguir incrementos de legitimidad; 4) proteger los logros producidos ante las variaciones fortuitas del entorno; 5) normalizar usos, procesos y costumbres; 6) reestructurar la organización y 7) mantener la calma ante las amenazas a la institución.

El neo-institucionalismo pone el foco en las instituciones, en sus reglas, procedimientos y sistemas culturales y, como enfoque teórico, nos permite estudiar las instituciones y la forma en la que estas se relacionan con su entorno. En este trabajo se considera que las instituciones incorporan procesos y formas de actuar con el objetivo de dar contestación a aquello que espera su entorno inmediato ${ }^{3}$. Este proceso se concreta en distintas estrategias marcadas por el isomorfismo coercitivo, el isomorfismo mimético y el isomorfismo normativo ${ }^{3}$ (DiMaggio y Powell, 1983; Meyer y Rowan, 1977; Peters, 2005).

${ }^{4}$ La definición de cada uno de estos mecanismos de cambio es la siguiente: a) el isomorfismo coercitivo que tiene su base en la presión legal y social; b) el isomorfismo mimético en el que se buscan otros modelos que se puedan adaptar a la organización y así hacer frente a la incertidumbre y; c) el isomorfismo normativo que hace referencia a los aspectos profesionales de un trabajo concreto (DiMaggio y Powell, 1983: 150).

${ }^{5}$ Una de las primeras críticas que se le ha realizado al Neo-Institucionalismo Sociológico es la falta de elección que se le presuponían a los individuos a la hora de tomar decisiones, situación que a partir de trabajos de autores como DiMaggio (1988) y Oliver (1991) parece que se haya corregido (Greenwood et al., 2008).

${ }^{6}$ El término de legitimidad definido por Suchman (1995) se puede definir de la siguiente manera: "[...] una percepción o suposición generalizada de que las acciones de una entidad son deseables, apropiadas o apropiadas dentro de un sistema socialmente construido de normas, valores, creencias y definiciones". 
${ }^{7}$ Una situación se exacerba con un pluralismo polarizado exacerbado en la que no existe una separación entre intereses económicos y de influencia hacia lo público y el trabajo periodístico (Hallin y Mancini, 2008).

8 Tal como se podrá comprobar en el siguiente subepígrafe.

${ }^{9}$ Somos conscientes de que este panorama mediático puede variar a lo largo del tiempo por la irrupción de las Web 2.0. No obstante, el papel de los medios de comunicación tiene un peso específico a corto y medio plazo que difícilmente podrá ser substituido en un futuro cercano.

${ }^{10}$ La traducción de esta cita de Kopecky y Mair (2011) es la siguiente: [...]Un recurso cada vez más valioso con el que las partes pueden tratar de asegurar su supervivencia y éxito organizativo en los sistemas políticos contemporáneos 


\section{(c) (i) (2) \\ BY SA \\ Licencia Creative Commons \\ Miguel Hernández Communication Journal \\ mhjournal.org}

\section{Cómo citar este texto:}

Gonzalo Pardo Beneyto y María Ángeles Abellán López (2017): "El proceso de cartelización en entornos institucionalizados: legitimación y comunicación política", en Miguel Hernández Communication Journal, nº, pp. 295 a 317. Universidad Miguel Hernández, UMH (Elche-Alicante). Recuperado el _ de del artículo en mhjournal.org] 
